\title{
Wave Techniques for Noise Modeling and Measurement
}

\author{
Scott W. Wedge, Member, IEEE, and David B. Rutledge, Senior Member, IEEE
}

\begin{abstract}
The noise wave approach is applied to analysis, modeling, and measurement applications. Methods are presented for the calculation of component and network noise wave correlation matrices. Embedding calculations, relations to twoport figures-of-merit, and transformations to traditional representations are discussed. Simple expressions are derived for MESFET and HEMT noise wave parameters based on a linear equivalent circuit. A noise wave measurement technique is presented and experimentally compared with the conventional method.
\end{abstract}

\section{INTRODUCTION}

$\mathrm{C}$

IRCUIT theory provides numerous alternatives for the characterization of noise in linear networks. Hartmann [1] alone presented twelve representations for the simple two-port. Noise is typically characterized using combinations of equivalent voltage and current sources. For high frequency circuit applications, however, a wave interpretation of noise has advantages. Noise waves have received a moderate amount of interest since their introduction by Penfield [2]. Bosma [3] used noise waves to solve the Haus and Adler [4] optimum noise performance problem. Hecken [5] used them to simplify noise performance calculations through signal-flow graph theory. Kanaglekar et al. [6] and Dobrowolski [7], [8] have presented CAD algorithms strictly in terms of noise waves and scattering parameters.

In the noise wave representation, a circuit element's noise is described using waves that emanate from its ports. A linear two-port represented by noise waves and scattering parameters is shown schematically in Fig. 1. Noise waves $c_{1}$ and $c_{2}$ contribute to the scattered waves such that the wave variables and scattering parameters satisfy

$$
\left(\begin{array}{l}
b_{1} \\
b_{2}
\end{array}\right)=\left(\begin{array}{ll}
s_{11} & s_{12} \\
s_{21} & s_{22}
\end{array}\right)\left(\begin{array}{l}
a_{1} \\
a_{2}
\end{array}\right)+\left(\begin{array}{l}
c_{1} \\
c_{2}
\end{array}\right) .
$$

The noise waves are time-varying complex random variables characterized by a correlation matrix $C_{s}$ given by

$$
C_{s}=\left(\begin{array}{ll}
\overline{\left|c_{1}\right|^{2}} & \overline{c_{1} c_{2}^{*}} \\
\overline{c_{2} c_{1}^{*}} & \overline{\left|c_{2}\right|^{2}}
\end{array}\right)
$$

Manuscript received December 9, 1991; revised March 31, 1992.

S. W. Wedge was with the Division of Engineering and Applied Science, California Institute of Technology. He is now with EEsof, Westlake Village, CA 91362-4020.

D. B. Rutledge is with the Division of Engineering and Applied Science California Institute of Technology, Pasadena, CA 91125.

IEEE Log Number 9292892.

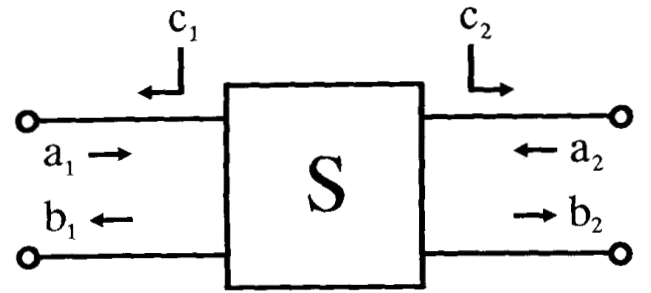

Fig. 1. The schematic representation of a two-port circuit element using scattering parameters and noise waves.

where the overbar indicates time averaging with an implicit assumption of ergodicity and jointly wide-sense stationary processes. The diagonal terms of $C_{s}$ give the noise power deliverable to the terminations in a $1-\mathrm{Hz}$ bandwidth. The off-diagonal terms are correlation products. The noise wave correlation matrix $C_{s}$ is Hermitian and its components are referred to as noise wave parameters.

The strength of the noise wave representation lies in a compatibility with distributed circuit variables that permits noise analysis problems to be formalized and solved using scattering parameters. Simple relations can often be found between a circuit element's scattering and noise wave parameters. The availability of accurate scattering parameter measurements then contributes to the accuracy of the noise analysis. Noise wave parameters are numerically stable. The reflections and resonances common in microwave circuits may cause voltage and current quantities to vary dramatically, while the limited range of noise wave quantities makes them ideal for CAD applications. The wave interpretation of noise has also lead to alternative noise parameter measurement methods [9], [10].

In this paper new applications of the noise wave representation in analysis, modeling, and measurement are presented. Section II presents noise wave analysis techniques. Noise calculations for interconnected multiport networks and two-port figures-of-merit are discussed. In Section III, equivalent circuits are used to derive simple expressions for the noise wave parameters of the MESFET and HEMT. The modeling approach taken is compared with experiment. Section IV describes a new method for making direct measurement of noise wave parameters. The method is simpler than conventional methods that use source-pull tuners to extract $T_{\min }, \Gamma_{\mathrm{opt}}$, and $R_{n}$. The noise wave and conventional measurement techniques are compared experimentally. 


\section{Noise Wave Network Analysis}

Analyzing a network for its noise properties involves its division into the smallest number of uncorrelated components. The signal and noise correlation matrices for the components are used to solve for those of the overall network. In general, the network and its components are multiport elements. In the noise wave representation each multiport element has scattering matrix $S$, incident and output wave vectors ( $\boldsymbol{a}$ and $\boldsymbol{b}$, respectively), and noise wave vector $c$ satisfying

$$
b=S a+c .
$$

The multiport noise wave correlation matrix $C_{s}$ of each element is given by

$$
C_{s}=\overline{c c^{\dagger}}
$$

where the dagger indicates the Hermitian conjugate, and the overbar the time averaged correlation product. At a given frequency the signal and noise properties of a linear $n$-port device are completely characterized by $n \times n$ scattering and noise wave correlation matrices. In Fig. 2, this approach is compared to the more traditional multiport admittance and impedance representations. Included in the comparison are circuit diagrams and matrix relations for voltage, current, and wave quantities. The use of multiple noise representations can sometimes lead to more efficient analyses [11]; transformations can be used to avoid a nonexistent representation, to simplify calculations, or to avoid singularities. The family of transformations between wave, impedance, and admittance representations is included in Fig. 2. Admittance and impedance matrices are assumed normalized.

\section{A. Embedded Multiport Networks}

Any linear noise analysis problem can be solved through one or more applications of the embedded network problem illustrated in Fig. 3. A noisy multiport subnetwork with scattering matrix $S$ is shown embedded in a noisy subnetwork with scattering matrix $\boldsymbol{T}$. The two subnetworks have known noise wave correlation matrices $\boldsymbol{C}_{\boldsymbol{s}}$ and $C_{t}$, respectively. The scattering and noise wave correlation matrices, $S_{\text {net }}$ and $C_{\text {net }}$, for the aggregate network are found by partitioning the embedding network's scattering matrix $\boldsymbol{T}$ into submatrices that satisfy

$$
\left(\begin{array}{c}
b_{e} \\
b_{i}
\end{array}\right)=\left(\begin{array}{cc}
T_{e e} & T_{e i} \\
T_{i e} & T_{i i}
\end{array}\right)\left(\begin{array}{l}
a_{e} \\
a_{i}
\end{array}\right)+\left(\begin{array}{c}
c_{e} \\
c_{i}
\end{array}\right)
$$

where subscript $i$ designates waves shared at the internal connections between networks $\boldsymbol{S}$ and $\boldsymbol{T}$, and subscript $\boldsymbol{e}$ designates the external waves at the $S_{\text {net }}$ terminals. The noise wave correlation matrix of network $T$ is similarly partitioned such that

$$
C_{t}=\left(\begin{array}{ll}
\overline{c_{e} c_{e}^{\dagger}} & \overline{c_{e} c_{i}^{\dagger}} \\
\overline{c_{i} c_{e}^{\dagger}} & \overline{c_{i} c_{i}^{\dagger}}
\end{array}\right) .
$$

The resulting noise wave correlation matrix is then given

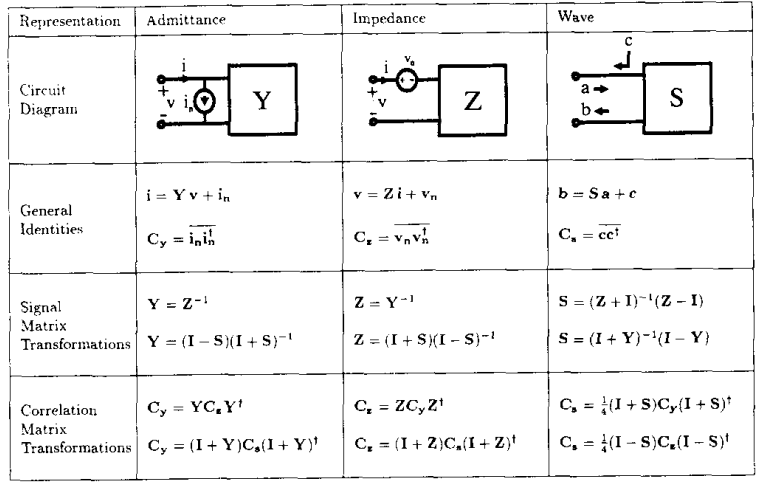

Fig. 2. A summary of the signal and noise representations and transformations for admittance, impedance, and wave parameters. The admittance matrix $Y$ and impedance matrix $Z$ are assumed to be normalized, and $I$ is the identity matrix.

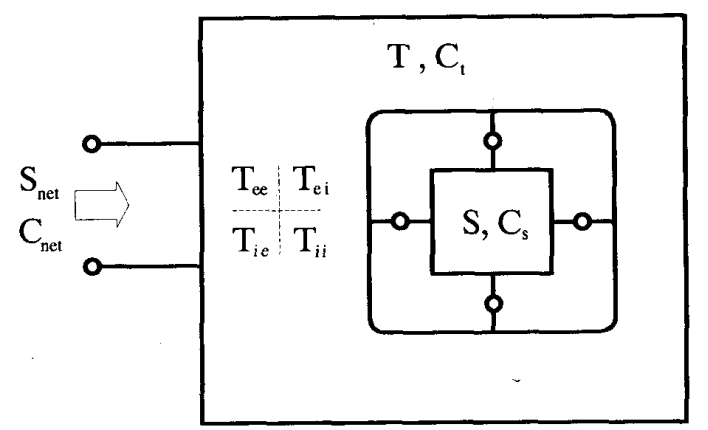

Fig. 3. A multiport network $S$ with noise wave correlation matrix $C_{s}$ embedded within network $T$ with correlation matrix $C_{t}$. The result of the embedding is scattering and correlation matrices $S_{\mathrm{net}}$ and $C_{\mathrm{net}}$. Network $T$ is partitioned in the manner shown, where subscript $e$ denotes reference to an external wave, and $i$ denotes reference to an internal wave. Internal waves are defined as those shared at the connections between $\boldsymbol{S}$ and $\boldsymbol{T}$.

by [3]

$$
C_{\text {net }}=\Lambda C_{s} \Lambda^{\dagger}+[I \mid \Lambda S] C_{t}[I \mid \Lambda S]^{\dagger}
$$

where the pipe symbol $(\mid)$ designates matrix augmentation, $\boldsymbol{I}$ is the identity matrix, and $\Lambda$ is the matrix given by

$$
\Lambda=T_{e i}\left(I-S T_{i i}\right)^{-1} .
$$

The network's scattering matrix is given by the well known expression [12]

$$
S_{\text {net }}=T_{e e}+\Lambda S T_{i e} .
$$

It is significant to note that matrix product $\boldsymbol{\Lambda} \boldsymbol{S}$ is commor: to both the $\boldsymbol{S}_{\text {net }}$ and $\boldsymbol{C}_{\text {net }}$ expressions. During analysis, the majority of computation time is spent on the matrix in version required for $\Lambda$, and for the calculation of this product. The penalty in combining a noise analysis with a deterministic analysis is therefore minimal. Since any network may be interpreted as a combination of embeddings, connection formulas (7) and (9) may be used as the basis for computer-aided analysis. Indeed, this approach is the wave equivalent of the admittance matrix CAD approach presented by Rizzoli and Lipparini [13]. 


\section{B. Two-Port Analysis}

Noise performance figures-of-merit are calculated from the signal and noise correlation matrices that result from a two-port network analysis. As with scattering parameters, noise waves are defined with respect to a normalizing impedance. This leads to simple expressions for amplifier noise performance when a zero source reflection coefficient $\left(\Gamma_{s}=0\right)$ is assumed. In terms of the scattering and noise wave parameters presented in (1) and (2), noise temperature $T_{n}$ is given by

$$
k T_{n}=\frac{\overline{\left|c_{2}\right|^{2}}}{\left|s_{21}\right|^{2}}
$$

where $k$ is Boltzmann's constant. Noise measure $M$ is written

$$
k T_{0} M=\frac{\overline{\left|c_{2}\right|^{2}}}{\left|s_{21}\right|^{2}+\left|s_{22}\right|^{2}-1}
$$

where $T_{0}$ is standard temperature $(290 \mathrm{~K})$. A $1-\mathrm{Hz}$ bandwidth is assumed in both expressions. Noise performance contours are generated by examining the variation of $T_{n}$ and $M$ with respect to non-zero $\Gamma_{s}$ values. In terms of the two-port scattering and noise wave correlation matrices, the noise temperature function is

$$
k T_{n}=\frac{\boldsymbol{\alpha} C_{s} \boldsymbol{\alpha}^{\dagger}}{\left(1-\left|\Gamma_{s}\right|^{2}\right)}
$$

where $\alpha$ is the $1 \times 2$ row matrix

$$
\boldsymbol{\alpha}=\left(\Gamma_{s} \frac{1-\Gamma_{s} s_{11}}{s_{21}}\right)
$$

and $C_{s}$ is the matrix given in (2). Noise measure may be written

$$
k T_{0} M=\frac{-\boldsymbol{\alpha} C_{s} \boldsymbol{\alpha}^{\dagger}}{\boldsymbol{\alpha}\left(\boldsymbol{I}-\boldsymbol{S} \boldsymbol{S}^{\dagger}\right) \boldsymbol{\alpha}^{\dagger}}
$$

where $S$ is the scattering matrix of the two-port. As before, the expressions for $T_{n}$ and $M$ assume a $1-\mathrm{Hz}$ bandwidth. The standard noise parameters $\Gamma_{\mathrm{opt}}, T_{\min }$, and $R_{n}$ may also be written in terms of the components of $C_{s}$. Equations for the conversion between these parameter sets are given in the Appendix.

\section{Component Modeling}

Prior to noise wave analysis, each component of a network must be represented by a scattering and noise wave correlation matrix. The extraction of scattering parameters is well understood, and noise wave correlation matrices may often be expressed in terms of these values. For the passive multiport with thermal noise, the noise wave correlation matrix is derived directly from the scattering matrix [14]

$$
\boldsymbol{C}_{\boldsymbol{s}}=k T\left(\boldsymbol{I}-\mathrm{SS}^{\dagger}\right)
$$

where $k$ is Boltzmann's constant and $T$ is the physical temperature. Combining this expression with (12) reveals the noise temperature of a passive two-port in terms of its scattering matrix:

$$
T_{n}=T \frac{\boldsymbol{\alpha}\left(\boldsymbol{I}-\boldsymbol{S} \boldsymbol{S}^{\dagger}\right) \boldsymbol{\alpha}^{\dagger}}{\left(1-\left|\Gamma_{s}\right|^{2}\right)} .
$$

Expressions comparable to (15) and (16) can be derived for active devices. Signal and noise modeling of the MESFET and HEMT are based on small-signal equivalent circuits and additional frequency-independent quantities proportional to noise correlation matrix values [15]-[20]. Pospieszalski [21] has shown that the frequency-independent quantities are tantamount to resistors in the equivalent circuit possessing effective temperatures. In the simplified intrinsic equivalent circuit of Fig. 4, the gatesource resistance $R_{g s}$ and drain-source resistance $R_{d s}$ are assigned equivalent temperatures $T_{g}$ and $T_{d}$, respectively. With the source grounded, noise wave $c_{1}$ emanates from the gate and $c_{2}$ from the drain. The value of $\overline{\left|c_{1}\right|^{2}}$ is due only to the temperature of input resistance $R_{g s}$ and is given by

$$
\overline{\left|c_{1}\right|^{2}}=k T_{g}\left(1-\left|s_{11}\right|^{2}\right) .
$$

Noise produced by $R_{g s}$ induces a voltage across $C_{g s}$ that is transferred to the drain via transconductance $g_{m}$. The result is the noise correlation term

$$
\overline{c_{2} c_{1}^{*}}=\frac{s_{21}}{s_{11}-1} \overline{\left|c_{1}\right|^{2}} .
$$

The output noise wave $c_{2}$ results from correlated noise from the gate, and noise generated by output resistance $R_{d s}$ at equivalent temperature $T_{d}$. It is given by

$$
\overline{\left|c_{2}\right|^{2}}=\frac{\left|s_{21}\right|^{2}}{\left|1-s_{11}\right|^{2}} \overline{\left|c_{1}\right|^{2}}+k T_{d}\left(1-\left|s_{22}\right|^{2}\right) \text {. }
$$

Expressions for the scattering and noise wave parameters in terms of the equivalent circuit values of Fig. 4 are given in the Appendix.

Accurate device signal and noise modeling generally requires a more detailed equivalent circuit than that of Fig. 4: stray capacitance, lead resistance and inductance must be added. The effects of additional elements on the scattering and correlation matrices are computed by applying embedding calculations (5)-(9); the equivalent circuit of Fig. 4 is considered embedded in a subcircuit consisting of the additional elements. The embedding circuit is typically passive with correlation matrix found from (15). A required intermediate step is a two-port to three-port conversion of $\boldsymbol{C}_{\boldsymbol{s}}$. This is achieved by recognizing that the elements of an indefinite $N$-port noise wave correlation matrix satisfy

$$
\sum_{j=1}^{N} \overline{c_{j} c_{k}^{*}}=\sum_{k=1}^{N} \overline{c_{j} c_{k}^{*}}=0
$$

as do the elements of an indefinite noise current correlation matrix.

This noise analysis procedure has been applied to the Fujitsu FSX02X MESFET and FHR02X HEMT using the 


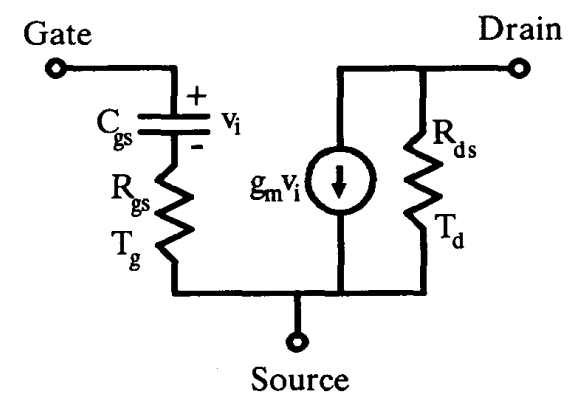

Fig. 4. Intrinsic equivalent circuit for MESFET and HEMT transistors. Gate temperature $T_{p}$ is the equivalent temperature of $R_{p s}$. Drain temperature $T_{d}$ is the equivalent temperature of $R_{d s}$. The noise voltage generated by $R_{p s}$ appears at $v_{i}$ resulting in correlation between input and output.

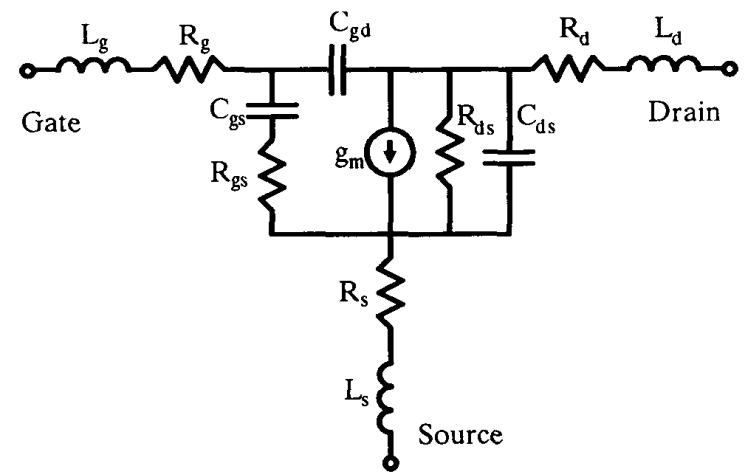

Fig. 5. A more accurate equivalent circuit for the chip form MESFET and HEMT. During noise analysis, the parasitic resistances $R_{g}, R_{s}$, and $R_{d}$ are assumed to be at standard temperature.

TABLE I

COMPONENT VAlues for Elements in THE EOUIVALENT CiRCUIT OF Fig. 5 USED TO MODEL THE FUJTSU FSX02X MESFET AND FHR02X HEMT

\begin{tabular}{lll}
\hline Parameter & FSX02X & FHR02X \\
\hline$g_{m 0}(\mathrm{mS})$ & 42.5 & 55 \\
$\tau(\mathrm{psec})$ & 2.0 & 0.85 \\
$C_{g s}(\mathrm{pF})$ & 0.33 & 0.2 \\
$C_{g d}(\mathrm{pF})$ & 0.033 & 0.025 \\
$C_{d s}(\mathrm{pF})$ & 0.115 & 0.049 \\
$R_{g s}(\Omega)$ & 3.5 & 2.5 \\
$R_{d s}(\Omega)$ & 270.0 & 188.7 \\
$R_{g}(\Omega)$ & 0.3 & 1.3 \\
$R_{s}(\Omega)$ & 1.8 & 1.3 \\
$R_{d}(\Omega)$ & 3.0 & 1.3 \\
$L_{g}(\mathrm{nH})$ & 0.12 & 0.1 \\
$L_{s}(\mathrm{nH})$ & 0.05 & 0.08 \\
$L_{d}(\mathrm{nH})$ & 0.12 & 0.1 \\
$T_{g}(\mathbf{K})$ & 290 & 290 \\
$T_{d}(\mathrm{~K})$ & 1375 & 1100 \\
\hline
\end{tabular}

The transconductance $g_{m}$ has an associated transit time $\tau$ such that $g_{n}=g_{m 0} e^{-j \omega \tau}$. Gate temperature $T_{g}$ is the equivalent temperature of $R_{y s}$. Drain temperature $T_{d}$ is the equivalent temperature of $R_{d s}$. Values for both devices are for $I_{d s}=10 \mathrm{~mA}$. The FSX02X values correspond to a bias voltage $V_{d s}=3 \mathrm{~V}$, while $V_{d s}=2 \mathrm{~V}$ for the FHR02X.

equivalent circuit of Fig. 5. Parameter values for the equivalent circuit are listed in Table I. The results have been converted to standard noise parameters (using the
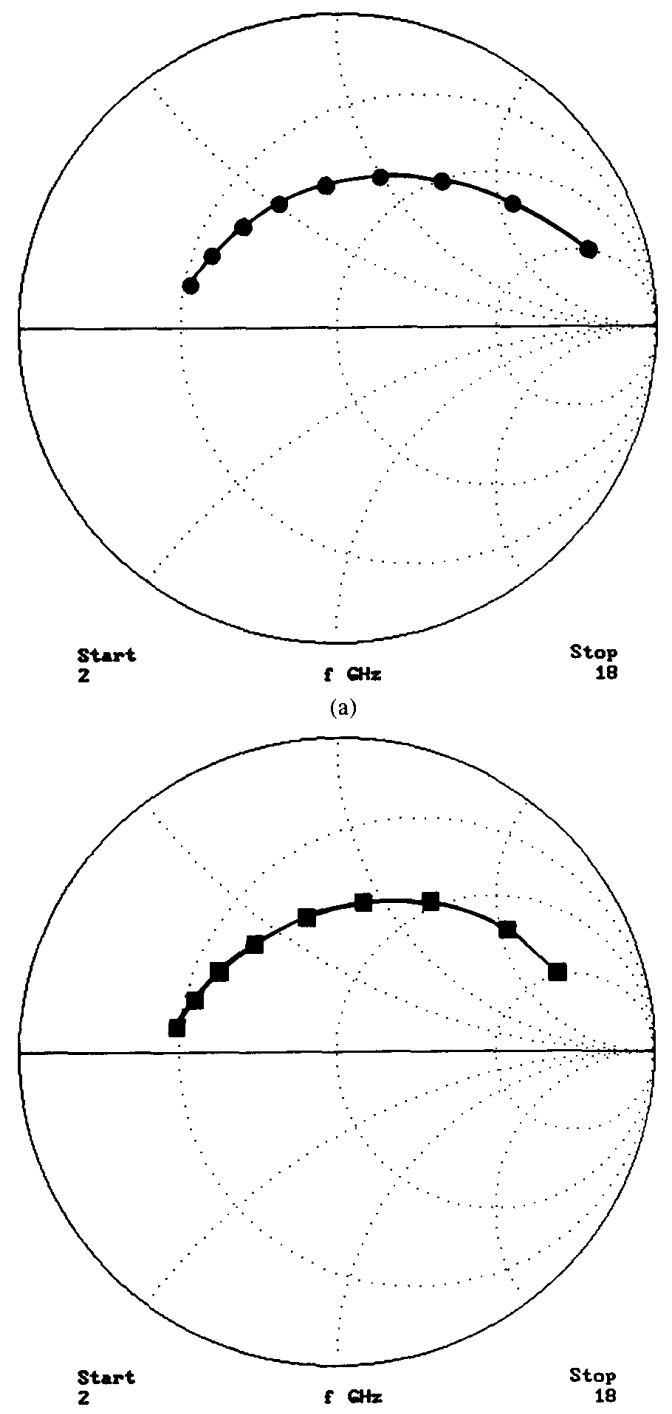

(b)

Fig. 6. Smith chart comparison of theory (a) and measurement (b) of optimum reflection coefficient $\Gamma_{\text {opt }}$ for the Fujitsu FSX02X MESFET. The theoretical plot was made with $T_{d}=1375 \mathrm{~K}$. Plots are from $2-18 \mathrm{GHz}$, running counterclockwise over frequency, with points shown in $2 \mathrm{GHz}$ increments.

equations found in the Appendix) for a comparison with measured data provided by the manufacturer. Given in Fig. 6 are Smith chart plots of theory and measurement for $\Gamma_{\text {opt }}$ for the Fujitsu FSX02X MESFET from 2-18 GHz. The theoretical plot assumes a standard gate temperature $T_{g}=290 \mathrm{~K}$, suggesting that this noise is thermal in nature. A large drain temperature value $\left(T_{d}=1375 \mathrm{~K}\right)$ is needed to represent all noise processes in the drain-source region. Theory and measurement for the noise figure minimum $F_{\min }$ and noise resistance $R_{n}$ are given in Fig. 7 . Noise figure minimum and $\Gamma_{\text {opt }}$ predictions are quite good. Noise resistance is slightly underestimated. Better $R_{n}$ and $F_{\text {min }}$ matching is possible by adding a pole to the fre- 


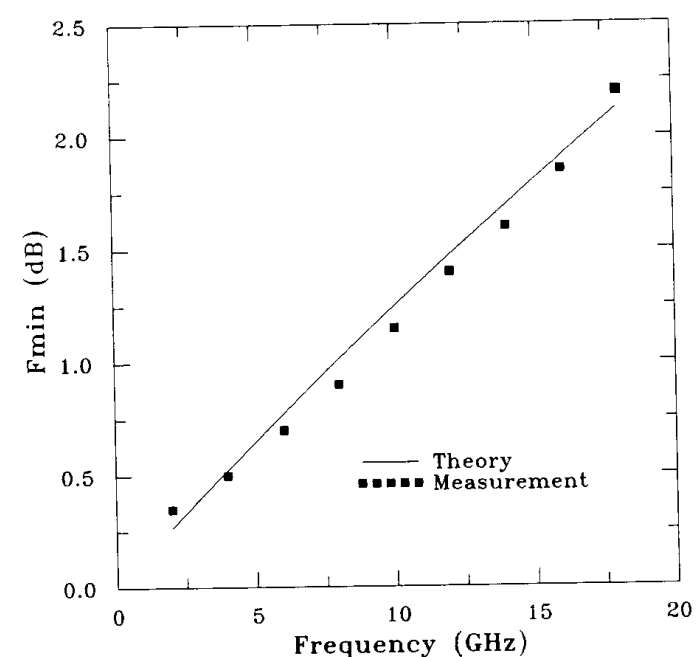

(a)

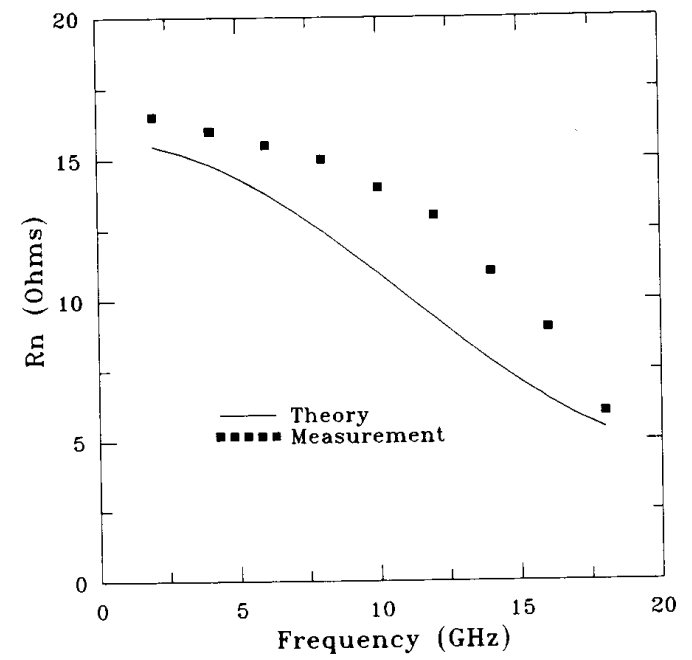

(b)

Fig. 7. Comparison of theory and measurement of noise figure minimum $F_{\min }\left(\right.$ a) and noise resistance $R_{n}($ b) for the Fujitsu FSX02X MESFET. The theoretical plot was made with $T_{d}=1375 \mathrm{~K}$.

quency behavior of $g_{m}$. Low frequency $F_{\min }$ predictions can be expected to deviate with measurements as $\left|\Gamma_{\text {opt }}\right|$ approaches unity. Shown in Fig. 8 are Smith chart plots of modeled and measured $\Gamma_{\text {opt }}$ for the Fujitsu FHR02X HEMT from 2-26 GHz. Standard temperature is again used for the gate. Graphs showing comparisons of $F_{\text {min }}$ and $R_{n}$ are shown in Fig. 9. As before, predictions are good with the exception of $R_{n}$ which deviates from theory at lower frequencies.

These examples demonstrate surprisingly good room temperature MESFET and HEMT noise modeling with a single parameter: drain temperature $T_{d}$. All other elements of the equivalent circuit (derived from scattering parameter measurements) were assumed to be at standard temperature. The limitations of this approach include de-

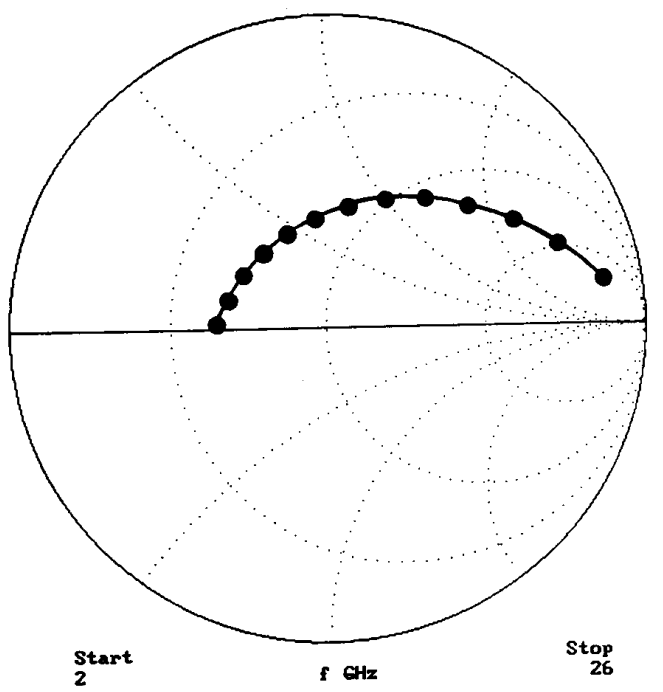

(a)

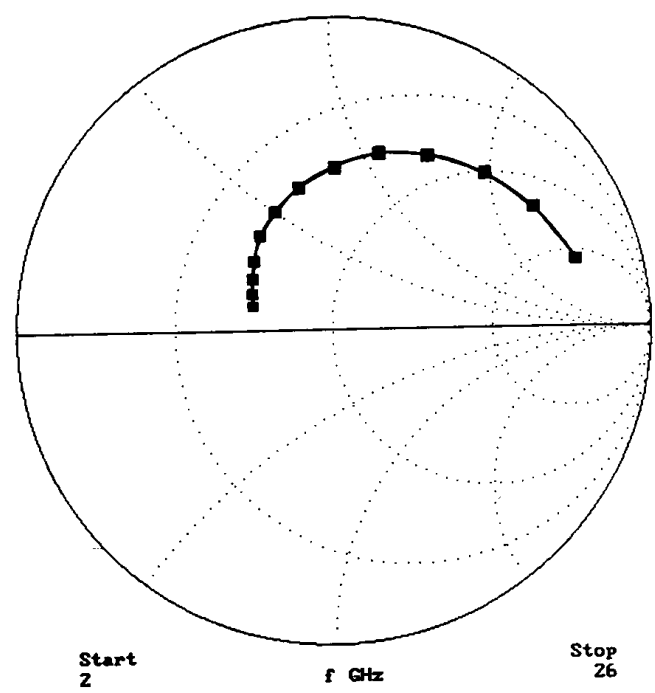

(b)

Fig. 8. Smith chart comparison of theory (a) and measurement (b) of optimum reflection coefficient $\Gamma_{\text {opt }}$ for the Fujitsu FHR02X HEMT. The theoretical plot was made with $T_{d}=1100 \mathrm{~K}$. Plots are from $2-26 \mathrm{GHz}$, running counterclockwise over frequency, with points shown in $2 \mathrm{GHz}$ increments.

pendence on the accuracy of the equivalent circuit and the lack of low frequency noise. Yet, it is possible to express $T_{d}$ as a function of frequency and bias conditions for broadband bias dependent noise modeling. The noise wave approach simplifies active and passive device modeling by using scattering parameters and these physical or equivalent temperature values.

\section{Noise Wave Measurements}

To measure the standard two-port noise parameters $T_{\min }, \Gamma_{\text {opt }}$, and $R_{n}$, a source-pull tuner is used to present various $\Gamma_{s}$ values to the device under test (DUT). The tuner complicates the measurement process. It may pos- 


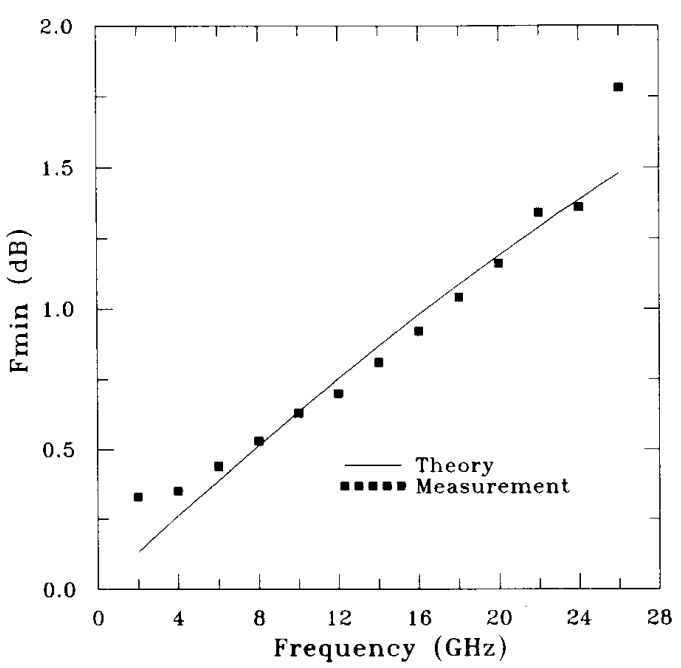

(a)

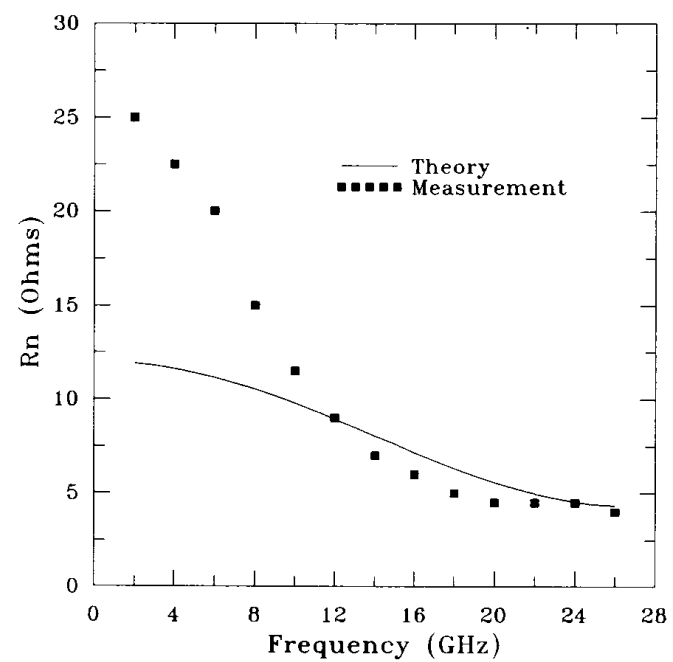

(b)

Fig. 9. Comparison of theory and measurement of noise figure minimum $F_{\min }\left(\right.$ a) and noise resistance $R_{n}$ (b) for the Fujitsu FHR02X HEMT. The theoretical plot was made with $T_{d}=1100 \mathrm{~K}$

sess repeatability error, have restricted tuning and frequency ranges, require frequent calibration, or cause low frequency oscillations in microwave transistors [19]. Direct measurement of the noise wave parameters $\left(\overline{\left|c_{1}\right|^{2}}\right.$, $\overline{c_{1} c_{2}^{*}}$, and $\overline{\left|c_{2}\right|^{2}}$ ) is a simpler process due to their definition with respect to a normalizing impedance.

The apparatus represented schematically in Fig. 10 has been used to measure noise wave parameters. The DUT is shown with scattering matrix $\boldsymbol{S}$ and emanating noise waves $c_{1}$ and $c_{2}$. At each port of the device, a circulator injects noise from a noise source into the device while terminating noise power that originates in the remainder of the system. Noise waves $c_{1}$ and $c_{2}$ emanate from the network and combine with scattered noise from the

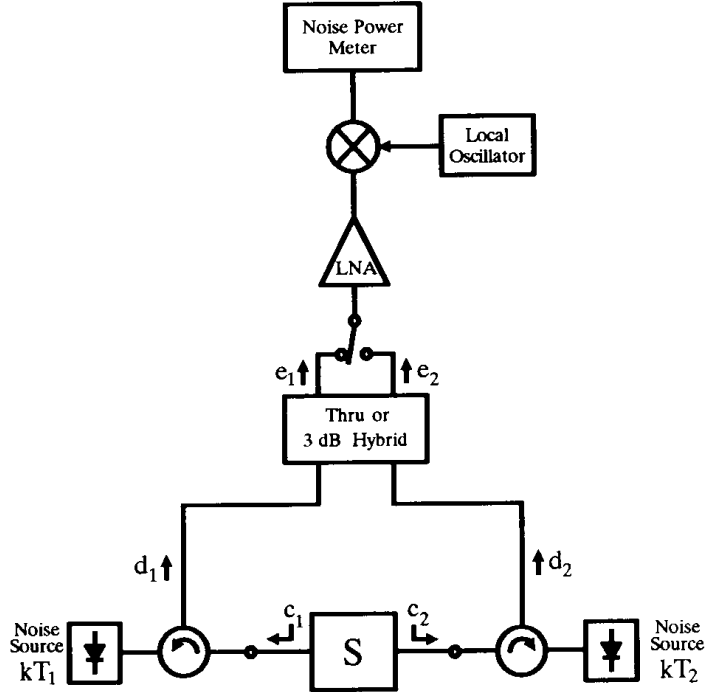

Fig. 10. Apparatus for the measurement of two-port noise wave parame-

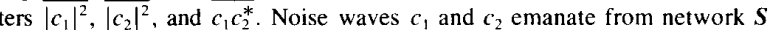
and combine with scattered waves from the noise sources to form waves $d$ and $d_{2}$. The noise sources have equivalent temperatures $T_{1}$ and $T_{2}$, each capable of taking on hot and cold values. At the center is placed either a "Thru" circuit or $3 \mathrm{~dB}$ hybrid. The "Thru" circuit is such that $d_{1}=e_{1}$ and $d_{2}$ and $e_{2}$ and permits direct measurement of $\left|c_{1}\right|^{2}$ and $\left|c_{2}\right|^{2}$. Substitution of a $0^{\circ} / 180^{\circ}$ hybrid allows measurement of $\operatorname{Re}\left(\overline{c_{1} c_{2}^{*}}\right)$, while a $90^{\circ}$ hybrid allows measurement of $\operatorname{Im}\left(\overline{c_{1} c_{2}^{*}}\right)$. The switch shown is assumed to be non-reflective. Noise power measurements are made at an intermediate frequency using an HP 8970 in noise power density mode.

sources to produce noise waves $d_{1}$ and $d_{2}$ given by

$$
\begin{aligned}
& d_{1}=c_{1}+\sqrt{k T_{1}} s_{11}+\sqrt{k T_{2}} s_{12} \\
& d_{2}=c_{2}+\sqrt{k T_{1}} s_{21}+\sqrt{k T_{2}} s_{22} .
\end{aligned}
$$

Power from the noise sources is written here in terms of effective temperatures $T_{1}$ and $T_{2}$, each capable of taking on known hot or cold values. Waves $d_{1}$ and $d_{2}$ will have measurable quantities

$$
\begin{aligned}
& \overline{\left|d_{1}\right|^{2}}=\overline{\left|c_{1}\right|^{2}}+k T_{1}\left|s_{11}\right|^{2}+k T_{2}\left|s_{12}\right|^{2} \\
& \overline{\left|d_{2}\right|^{2}}=\overline{\left|c_{2}\right|^{2}}+k T_{1}\left|s_{21}\right|^{2}+k T_{2}\left|s_{22}\right|^{2} \\
& \overline{d_{1} d_{2}^{*}}=\overline{c_{1} c_{2}^{*}}+k T_{1} s_{11} s_{21}^{*}+k T_{2} s_{12} s_{22}^{*} .
\end{aligned}
$$

The apparatus of Fig. 10 allows each of the above unknown right-hand-side values to be obtained through a set of noise power measurements. First, a "Thru" circuit is inserted at the center of the apparatus such that $d_{1}=e_{1}$ and $d_{2}=e_{2}$. Four noise power measurements are made of $\overline{\left|d_{1}\right|^{2}}$ and $\overline{\left|d_{2}\right|^{2}}$ using hot and cold values for both $T_{1}$ and $T_{2}$. Using (23) and (24), this measurement data is sufficient to solve for the six unknowns $\left|c_{1}\right|^{2},\left|c_{2}\right|^{2},\left|s_{11}\right|^{2}$, $\left|s_{12}\right|^{2},\left|s_{21}\right|^{2}$, and $\left|s_{22}\right|^{2}$. Measurement of noise wave correlation can be accomplished with $3 \mathrm{~dB}$ hybrid couplers [14]. With a lossless $0^{\circ} / 180^{\circ} 3 \mathrm{~dB}$ hybrid inserted such 
that

$$
\begin{aligned}
& e_{1}=\frac{1}{\sqrt{2}}\left(d_{1}+d_{2}\right) \\
& e_{2}=\frac{1}{\sqrt{2}}\left(d_{1}-d_{2}\right)
\end{aligned}
$$

the measured noise powers are given by

$$
\begin{aligned}
& \overline{\left|e_{1}\right|^{2}}=\frac{1}{2}\left[\overline{\left|d_{1}\right|^{2}}+\overline{\left|d_{2}\right|^{2}}+2 \operatorname{Re}\left(\overline{d_{1} d_{2}^{*}}\right)\right] \\
& \overline{\left|e_{2}\right|^{2}}=\frac{1}{2}\left[\overline{\left|d_{1}\right|^{2}}+\overline{\left|d_{2}\right|^{2}}-2 \operatorname{Re}\left(\overline{d_{1} d_{2}^{*}}\right)\right] .
\end{aligned}
$$

The difference in these values yields

$$
\begin{aligned}
\overline{\left|e_{1}\right|^{2}}-\overline{\left|e_{2}\right|^{2}}= & 2 \operatorname{Re}\left(\overline{d_{1} d_{2}^{*}}\right) \\
= & 2\left[\operatorname{Re}\left(\overline{c_{1} c_{2}^{*}}\right)+k T_{1} \operatorname{Re}\left(s_{11} s_{21}^{*}\right)\right. \\
& \left.+k T_{2} \operatorname{Re}\left(s_{12} s_{22}^{*}\right)\right]
\end{aligned}
$$

where the values for $\overline{\left|e_{1}\right|^{2}}$ and $\overline{\left|e_{2}\right|^{2}}$ have been compared assuming consistent values of $T_{1}$ and $T_{2}$. The set of measurements taken at hot and cold values for $T_{1}$ and $T_{2}$ now results in solutions for unknowns $\operatorname{Re}\left(c_{1} c_{2}^{*}\right), \operatorname{Re}\left(s_{1\}} s_{21}^{*}\right)$, and $\operatorname{Re}\left(s_{12} s_{22}^{*}\right)$. When the $0^{\circ} / 180^{\circ}$ hybrid is replaced with a $90^{\circ} 3 \mathrm{~dB}$ hybrid, the difference in noise powers becomes

$$
\begin{aligned}
\overline{\left|e_{1}\right|^{2}}-\overline{\left|e_{2}\right|^{2}}= & 2 \operatorname{Im}\left(\overline{d_{1} d_{2}^{*}}\right) \\
= & 2\left[\operatorname{Im}\left(\overline{c_{1} c_{2}^{*}}\right)+k T_{1} \operatorname{Im}\left(s_{11} s_{21}^{*}\right)\right. \\
& \left.+k T_{2} \operatorname{Im}\left(s_{12} s_{22}^{*}\right)\right]
\end{aligned}
$$

and an additional set of measurements now permits solution of the imaginary components $\operatorname{Im}\left(c_{1} c_{2}^{*}\right), \operatorname{Im}\left(s_{11} s_{21}^{*}\right)$, and $\operatorname{Im}\left(s_{12} s_{22}^{*}\right)$.

This system has been constructed and a series of measurements performed at $4 \mathrm{GHz}$ on a MESFET amplifier over a range of bias currents. An HP 8970 noise figure meter (in power density mode) was used to measure noise power. A simple short-open-thru calibration procedure was followed to compensate for losses and noise in the apparatus. For experimental comparison, measurements were made at the same bias points using the conventional source-pull tuner technique. A mechanical slide-screw turner was used and the additional measurements and corrections recommended by Strid [22] were performed. The results, in terms of standard noise parameters, are compared in Fig. 11. The similarities are surprising considering the dramatic differences in the measurement methods and their sources of error: the tuner method has errors due to tuner repeatability, unstable bias, and noise figure measurement uncertainty; wave method errors are due to uncertainty in temperatures $T_{1}$ and $T_{2}$ and a simplified calibration procedure (the directional couplers were assumed to have ideal phase shifts).

The wave method of noise parameter measurement has several advantages. It uses many off-the-shelf compo-
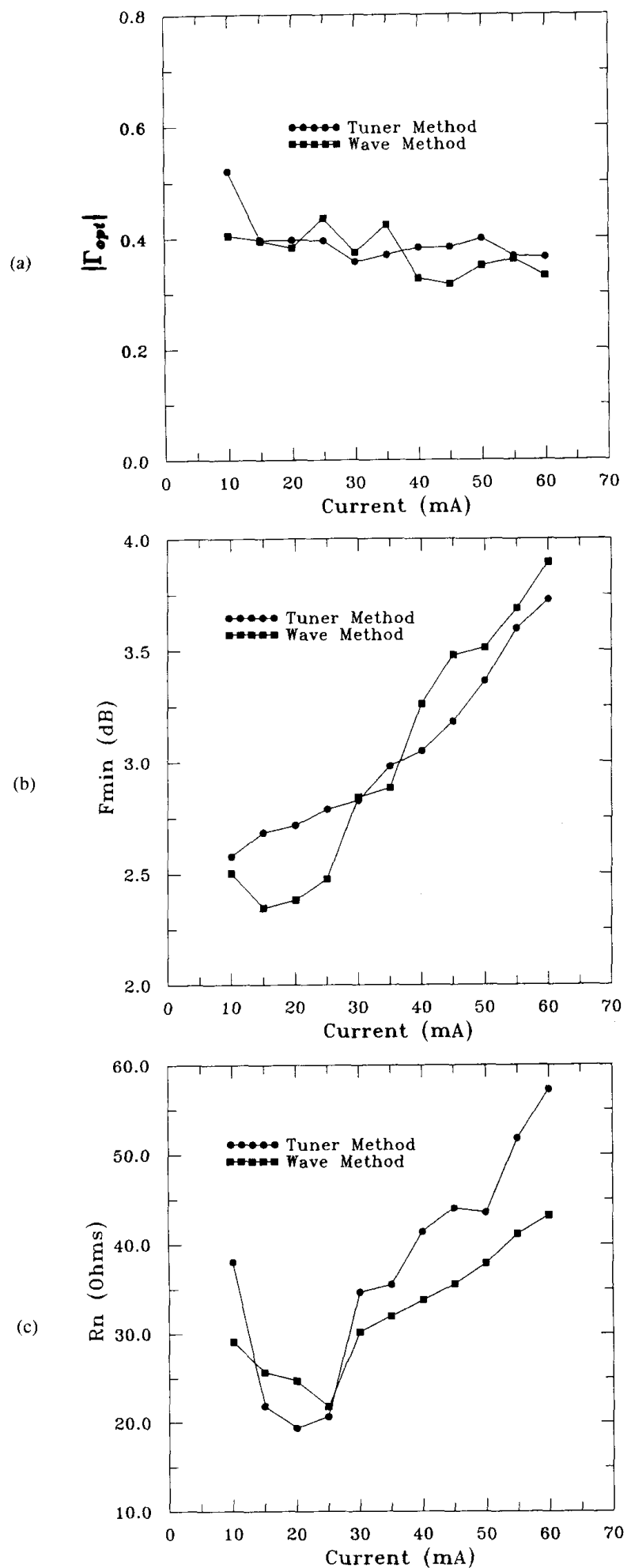

Fig. 11. Comparison of the tuner and wave measurement methods in determining the optimum reflection coefficient $\Gamma_{\mathrm{opt}}$ (a), the noise figure minimum $F_{\text {min }}$ (b), and the noise resistance $R_{n}$ (c) of an amplifier based on single Fujitsu FSC10 MESFET. The measurements were performed at 4 $\mathrm{GHz}$ with amplifier bias current varied from 10-60 mA. 
nents, may be configured for operation at RF through millimeter-wave frequencies, and requires no source-pull tuner. In addition to the noise wave parameters, the magnitudes of the scattering parameters, and the complex scattering parameter products $s_{11} s_{21}^{*}$ and $s_{12} s_{22}^{*}$ are determined. The system of equations that is manipulated is over-determined, and lends itself to statistical analysis. Noise contributions from the measurement apparatus are predicted by embedding equation (7), and may be removed by a calibration procedure. Many other configurations of the measurement apparatus of Fig. 10 are possible. Withington [10], for example, has presented a similar system that uses interferometric measurements. Measurement accuracy and bandwidth may be improved by using two LNA's and mixers. The directional coupler substitutions described here are avoided by using the sixport network described by Engen [23] as a single correlation network [24].

\section{CONCLUSIONS}

The noise wave representation offers alternative analysis, modeling and measurement techniques. Noise wave analysis may be performed solely in terms of distributed circuit variables. Derivation of noise wave parameters for many microwave components is a straightforward process, requiring only scattering parameters and physical or effective temperatures. The wave approach to noise parameter measurement offers advantages over conventional methods. It requires no source-pull tuner, uses off-theshelf components, and is promising for millimeter-wave applications.

\section{APPENDIX}

Noise wave parameters for active devices may be derived from the standard noise parameters or calculated from equivalent circuit values. In terms of $T_{\min }, \Gamma_{\mathrm{opt}}$, and $R_{n}$ the noise wave parameters are

$$
\begin{aligned}
& \overline{\left|c_{1}\right|^{2}}=k T_{\min }\left(\left|s_{11}\right|^{2}-1\right)+\frac{k t\left|1-s_{11} \Gamma_{\mathrm{opt}}\right|^{2}}{\left|1+\Gamma_{\mathrm{opt}}\right|^{2}} \\
& \overline{\left|c_{2}\right|^{2}}=\left|s_{21}\right|^{2}\left(k T_{\mathrm{min}}+\frac{k t\left|\Gamma_{\mathrm{opt}}\right|^{2}}{\left|1+\Gamma_{\mathrm{opt}}\right|^{2}}\right) \\
& \overline{c_{1} c_{2}^{*}}=\frac{-s_{21}^{*} \Gamma_{\mathrm{opt}}^{*} k t}{\left|1+\Gamma_{\mathrm{opt}}\right|^{2}}+\frac{s_{11}}{s_{21}} \overline{\left|c_{2}\right|^{2}}
\end{aligned}
$$

where $k t$ is the normalized temperature-energy given by

$$
k t=\frac{4 k T_{0} R_{n}}{Z_{0}}
$$

and $Z_{0}$ is the normalization impedance. The inverse relations are

$$
\begin{aligned}
k t & =\overline{\left|c_{1}-c_{2}\left(\frac{1+s_{11}}{s_{21}}\right)\right|^{2}} \\
k T_{\text {min }} & =\frac{\overline{\left|c_{2}\right|^{2}}-\overline{\left|c_{1} s_{21}-c_{2} s_{11}\right|^{2}}\left|\Gamma_{\mathrm{opt}}\right|^{2}}{\left|s_{21}\right|^{2}\left(1+\left|\Gamma_{\mathrm{opt}}\right|^{2}\right)}
\end{aligned}
$$

$$
\Gamma_{\mathrm{opt}}=\frac{\eta}{2}\left(1-\sqrt{1-\frac{4}{|\eta|^{2}}}\right)
$$

where

$$
\eta=\frac{\overline{\left|c_{2}\right|^{2}}+\overline{\left|c_{1} s_{21}-c_{2} s_{11}\right|^{2}}}{\overline{\left|c_{2}\right|^{2}} s_{11}-\overline{c_{1} c_{2}^{*}} s_{21}} .
$$

The inverse relations have been somewhat simplified through use of the identity

$$
\begin{aligned}
& \overline{\left|c_{1} \alpha-c_{2} \beta\right|^{2}} \\
& =\overline{\left|c_{1}\right|^{2}}|\alpha|^{2}-2 \operatorname{Re}\left(\overline{c_{1} c_{2}^{*}} \alpha \beta^{*}\right)+\overline{\left|c_{2}\right|^{2}}|\beta|^{2} .
\end{aligned}
$$

Noise wave parameters for the intrinsic MESFET and HEMT with the equivalent circuit given by Fig. 4 may be written in terms of scattering parameters or equivalent circuit parameters. The scattering matrix of the equivalent circuit of Fig. 4 is given by

$$
S=\left(\begin{array}{lc}
\frac{1+j \omega C_{g s}\left(r_{g s}-Z_{0}\right)}{1+j \omega C_{g s}\left(r_{g s}+Z_{0}\right)} & 0 \\
\frac{-2 g_{m} r_{d s} Z_{0}}{\left(r_{d s}+Z_{0}\right)\left[1+j \omega C_{g s}\left(r_{g s}+Z_{0}\right)\right]} & \frac{r_{d s}-Z_{0}}{r_{d s}+Z_{0}}
\end{array}\right)
$$

Solving for the noise wave parameters using (17)-(19) and assuming constant values of gate temperature $T_{g}$ and drain temperature $T_{d}$ gives

$$
\begin{aligned}
\overline{\left|c_{1}\right|^{2}}= & \frac{4 k T_{g} r_{g s} Z_{0} \omega^{2} C_{g s}^{2}}{1+\omega^{2} C_{g s}^{2}\left(r_{g s}+Z_{0}\right)^{2}} \\
\overline{c_{2} c_{1}^{*}}= & \frac{-j 4 k T_{g} r_{g s} r_{d s} Z_{0} g_{m} \omega C_{g s}}{\left(r_{d s}+Z_{0}\right)\left[1+\omega^{2} C_{g s}^{2}\left(r_{g s}+Z_{0}\right)^{2}\right]} \\
\overline{\left|c_{2}\right|^{2}}= & \frac{4 k T_{g} r_{g s} r_{d s} Z_{0} g_{m}^{2}}{\left(r_{d s}+Z_{0}\right)\left[1+\omega^{2} C_{g s}^{2}\left(r_{g s}+Z_{0}\right)^{2}\right]} \\
& +\frac{4 k T_{d} r_{d s} Z_{0}}{\left(r_{d s}+Z_{0}\right)^{2}} .
\end{aligned}
$$

These parameters are well behaved down to $\omega=0$, but low-frequency noise effects are not included. Frequency dependent expressions for $T_{g}$ and $T_{d}$ may be used to include these effects.

\section{REFERENCES}

[1] K. Hartmann, "Noise characterization of linear circuits," IEEE Trans. Circuits Syst., vol. CAS-23, pp. 581-590, Oct. 1976

[2] P. Penfield, "Wave representation of amplifier noise," IRE Trans. Circuit Theory, vol. CT-9, pp. 84-86, Mar. 1962.

[3] H. Bosma, "On the theory of linear noisy systems," Philips Res. Repts. Suppl., no. 10, 1967

[4] H. A. Haus and R. B. Adler, "Optimum noise performance of linear amplifiers," Proc. IRE, vol. 46, pp. 1517-1533, Aug. 1958.

[5] R. P. Hecken, "Analysis of linear noisy two-ports using scattering 
waves," IEEE Trans. Microwave Theory Tech., vol. MTT-29, pp. 997-1004, Oct. 1981

[6] N. G. Kanaglekar, R. E. McIntosh, and W. E. Bryant, "Wave analysis of noise in interconnected multiport networks," IEEE Trans. Microwave Theory Tech., vol. MTT-35, pp. 112-115, Feb. 1987.

[7] J. A. Dobrowolski, "A CAD-oriented method for noise figure com putation of two-ports with any internal topology, " IEEE Trans. Microwave Theory Tech., vol. MTT-37, pp. 15-20, Jan. 1989

[8] J. A. Dobrowolski, "Noise power sensitivities and noise figure minimization of two-ports with any internal topology," IEEE Trans. Microwave Theory Tech., vol. MTT-39, pp. 136-140, Jan. 1991.

[9] R. P. Meys, "A wave approach to the noise properties of linear microwave devices," IEEE Trans. Microwave Theory and Tech., vol. vol. MTT-26. pp. 34-37, Jan. 1978.

[10] S. Withington, "Scattered noise waves in microwave and $\mathrm{mm}$-wave networks." Microwave J.. vol. 32, pp. 169-178, June 1989.

[11] H. Hillbrand and P. H. Russer, "An efficient method for computer aided noise analysis of linear amplifier networks," IEEE Trans. Circuits Syst, vol. CAS-23, pp. 235-238, Apr. 1976.

[12] T. T. Ha, Solid State Microwave Amplifier Design. New York: Wiley, 1981.

[13] V. Rizzoli and A. Lipparini, "Computer-aided noise analysis of linear multiport networks of arbitrary topology," IEEE Trans. Microwave Theory Tech., vol. MTT-33, pp. 1507-1512, Dec. 1985.

[14] S. W. Wedge and D. B. Rutledge, "Noise waves and passive linear multiports," IEEE Microwave Guided Wave Lett., vol. 1, pp. 117119. May 1991.

[15] H. Statz, H. A. Haus, and R. A. Pucel "Noise characteristics of gallium arsenide field-effect transistors," IEEE Trans. Electron Devices, vol. ED-21, pp. 549-562, Sept. 1974.

[16] H. Fukui, "Design of microwave GaAs MESFET's for broad-band low-noise amplifiers," IEEE Trans. Microwave Theory Tech., vol. MTT-27, pp. 643-650, July 1979.

[17] A. F. Podell, "A functional GaAs FET noise model," IEEE Trans. Electron Devices, vol. ED-28, pp. 511-517, May 1981.

[18] M. S. Gupta, O. Pitzalis, S. E. Rosenbaum, and P. T. Greiling, "Microwave noise characterization of GaAs MESFET's: evaluation by on-wafer low-frequency output noise current measurement," IEEE Trans. Microwave Theory Tech., vol. MTT-35, pp. 1208-1218, Dec. 1987.

[19] A. Cappy, "Noise modeling and measurement techniques," IEEE Trans. Microwave Theory Tech., vol. 36, pp. 1-10, Jan. 1988.

[20] R. K. Froelich, "An improved model for noise characterization of microwave GaAs FET's," IEEE Trans. Microwave Theory Tech., vol. 38, pp. 703-706, June 1990.
121] M. W. Pospieszalski "Modeling of noise parameters of MESFET's and MODFET's and their frequency and temperature dependence,' IEEE Trans. Microwave Theory Tech., vol. 37, pp. 1340-1350, Sept 1989

[22] E. W. Strid, "Measurement of losses in noise-matching networks," IEEE Trans. Microwave Theory Tech., vol. MTT-29, pp. 247-252, Mar. 1981.

[23] G. F. Engen, "An improved circuit for implementing the six-por technique of microwave measurements," IEEE Trans. Microwave Theory Tech., vol. MTT-25, pp. 1080-1083, Dec. 1977.

124] S. W. Wedge, "Computer-aided design of low noise microwave circuits," Ph.D. dissertation, California Institute of Technology, 1991.

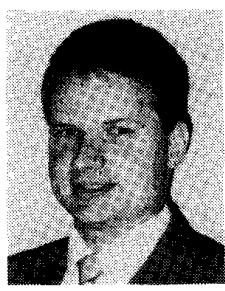

Scott W. Wedge (S'82-M'90) received the B.S degree from the California State Polytechnic Uni versity in 1983, the M.S. degree from the Uni. versity of Illinois in 1986, and the Ph.D. degree from the Califomia Institute of Technology in 1991, all in electrical engineering.

From 1983 to 1991 he was with Hughes Air craft Company, Ground Systems Group, Fuller ton, $\mathrm{CA}$, and is now with EEsof, Westlake Vil lage, CA. His interests and experience from industry and academia include RF and MMIC circuit design, computational electromagnetics, microwave measurement systems, and microwave $\mathrm{CAD}$.

Dr. Wedge is a member of Tau Beta $\mathrm{Pi}$ and Eta Kappa $\mathrm{Nu}$, a registered professional engineer in California, and a former Hughes Doctoral Fellow He is co-author of the educational microwave CAD program, Puff, which has over 8000 users worldwide.

David B. Rutledge (M'75-SM'89), for a photograph and biography, see this issue, p. 2003 